The published manuscript can be found here: DOI: 10.1007/s00426-016-0818-6

\title{
Cue-target contingencies modulate voluntary orienting of spatial attention: Dissociable effects for speed and accuracy
}

\author{
Mario Bonato ${ }^{1,2 \S} *$, Matteo Lisi ${ }^{3,2 \S}$, Sara Pegoraro $^{2}$, Gilles Pourtois ${ }^{4}$ \\ ${ }^{1}$ Department of Experimental Psychology, Ghent University, Belgium \\ ${ }^{2}$ Department of General Psychology, University of Padova, Italy \\ ${ }^{3}$ Laboratoire Psychologie de la Perception (CNRS UMR 8242), Universitè Paris-Descartes, Paris, \\ France \\ ${ }^{4}$ Department of Experimental Clinical and Health Psychology, Ghent University, Belgium \\ $\S=$ Equal contribution
}

\section{Keywords:}

spatial attention; attentional orienting; cue predictivity; proportion valid; endogenous; cue-target contingencies

Word Count: approximately 5700 (body)

Running head: Contingencies learning and spatial orienting

\section{*Corresponding author:}

Dr Mario Bonato,

Department of Experimental Psychology,

Henri Dunantlaan 2, B9000, Ghent, Belgium

e-mail: mario.bonato@ugent.be 


\section{Abstract}

Voluntary orienting of spatial attention is typically investigated by visually presented directional cues, which are called predictive when they indicate where the target is more likely to appear. In this study we investigated the nature of the potential link between cue predictivity (the proportion of valid trials) and the strength of the resulting, covert, orienting of attention. Participants judged the orientation of a unilateral Gabor grating preceded by a centrally-presented, non-directional, colour cue, arbitrarily prompting a leftwards or rightwards shift of attention. Unknown to them, cue predictivity was manipulated across blocks, whereby the cue was only predictive for either the first or the second half of the experiment. Our results show that the cueing effects were strongly influenced by the change in predictivity. This influence differently emerged in response speed and accuracy. The speed difference between valid and invalid trials was significantly larger when cues were predictive, and the amplitude of this effect was modulated at the single trial level by the recent trial history. Complementary to these findings, accuracy revealed a robust effect of block history and also a different time-course compared to speed, as if it mainly mirrored voluntary processes. These findings, obtained with a new manipulation and by using arbitrary non-directional cueing, demonstrate that cue-target contingencies strongly modulate the way attention deploys in space. 
The commonsense intuition suggests the existence of specific cognitive mechanisms allowing to voluntarily shift spatial attention in the environment towards potentially relevant stimuli. These mechanisms have been thoroughly scrutinized in the past. The standard method to isolate the voluntary (endogenous) component of spatial attention is the Posner's (1980) cuing paradigm, whereby leftward/rightward oriented arrow cues with a predictive value are centrally presented before the appearance of a unilateral target. Cues can be either valid (target appearing at the cued location) or invalid (target not appearing at the cued location). For a long time, the orienting of spatial attention arising from central arrows has been conceived as a mechanism involving pure voluntary control of attention, occurring only if the arrows were predictive, that is if they indicated with above-than-chance frequency the spatial position of the upcoming target (e.g. Muller $\&$ Rabbit, 1989; Posner, Walker, Friedrich, \& Rafal, 1984). This approach is consistent with Jonides (1981)'s original statement postulating that the endogenous orienting of spatial attention is primarily sensitive to the expectations of the participants.

Some subsequent studies reported discrepant results without challenging the assumption that predictive cues are necessary to induce endogenous shifts of spatial attention. Among them, Tassinari, Aglioti, Chelazzi, Marzi and Berlucchi (1987) showed that attention can be voluntarily allocated without using predictive cueing (see also Koshino, Warner, \& Juola, 1992).

At the end of the 90's, the use of symbolic, directional cues (e.g. arrows) was criticized. Once more, this occurred without focusing on the effects of cue predictivity. Several studies (Eimer, 1997; Hommel et al., 2001; Tipples, 2002; Ristic \& Kingstone, 2006) clarified that centrally-presented arrows induce a specific orienting of spatial attention, termed "reflexive" because occurring although participants know that there is no benefit in orienting towards the cued location. This type of orienting shares some characteristics with the "genuine" exogenous orienting of attention that is triggered by using peripheral and non predictive cues. Consistent with this assumption, in patients with unilateral spatial neglect, it is plausible that involuntary components mostly contribute to the characteristic disengage deficit for contralesional targets (Bonato, Priftis, Marenzi \& Zorzi, 2009; Olk, Hildebrandt, \& Kingstone, 2010). However, as already outlined here above, past studies primarily questioned the use of asymmetric symbolic, directional cues as arrows to induce endogenous spatial orienting (Lambert, Roser, Wells, \& Heffer, 2006) without studying the role of cue predictivity. Nevertheless, indirect evidence suggests that predictivity might interact with expectations. Cueing effects (performance for invalid vs valid trials) by predictive (central) arrows have been described to greatly exceed the sum of the so-called reflexive orienting of spatial attention (triggered by non-predictive arrows), and of the "volitional" shift of spatial attention (triggered by predictive non-directional cues, Ristic \& Kingstone, 2006). 
In sum, a common assumption in the literature is that voluntary shifts of spatial attention can be induced only when the cue reliably predicts target's position(Carrasco, 2011; Prinzmetal \& Landau, 2008), and yet not much is known about the effects of cue predictivity per se. The main goal of our study was to explore the role of cue predictivity in spatial orienting in the absence of confounding factors like explicit symbolic value/physical asymmetry of the cues. To this aim, we systematically manipulated, unbeknown to participants, cue predictivity in a specifically designed variant of the Posner task. Participants were required to orient attention towards the left or on the right of the fixation point according to a centrally presented, color cue which, unlike arrows, did not automatically prompt any directional shift of spatial attention. This feature is of paramount importance because it resulted in an arbitrary association between cue identity and target position. We decided to use a "short" vs a "long" SOA to capture the "fast" exogenous and the "slow" endogenous attentional processes, respectively. Moreover, we analyzed the data using linear and generalized linear mixed-effects models, and we capitalized on the possibility they offer to perform single trial data analysis to model, for instance, effects of (immediately) preceding trials on the current one (Kinoshita, Mozer \& Forster, 2011).

Our new manipulation was designed to highlight potential modulatory effect of cue predictivity but also to test whether, when cues are fully non-directional and do not carry any overlearned symbolic meaning, predictivity is a necessary condition for a reliable orienting of spatial attention to occur. Computer-based assessment is a very sensitive option (compared to paper and pencil tests) for detecting spatial awareness deficits after brain damage (Bonato, 2015). However, the use of predictive cues remains the most frequently adopted methodology to specifically quantify impairments in voluntary orienting of spatial attention after brain-damage (Losier \& Klein, 2001). Similarly, it is still the gold standard approach for exploring the neural correlates of endogenous spatial attention (Corbetta \& Schulman, 2011). Does predictivity (cue-target contingencies) add some automatic, associative/reflexive components to spatial orienting? If this would be the case, then it could potentially challenge the dichotomy between automatic and voluntary components of attention at least in the way it is typically assessed in neuroimaging and in clinical neuropsychology. 


\section{Method}

\section{Overview}

The task was based on the well-established Posner paradigm. Lateralized targets were presented after a cue indexing where attention had to be oriented "covertly", that is without overt eye movements. In order to obtain a more fine-grained picture of the effects of the proportion of valid trials, we opted for the use of a discrimination task, allowing to analyze both Reaction Times (RTs) and accuracy data. With such a manipulation, attention orienting is thought to be slower than in simple detection tasks (Rafal \& Henik, 1994) and cueing effects are usually more reliable (Chica, Martín-Arévalo, Botta \& Lupiánez, 2014). Two specific manipulations, yet never combined together in earlier studies, were implemented. First, non-directional cues were used (colors). Second, predictivity (that is the proportion of valid trials, namely how often the target actually appeared at the cued location) was varied across the experimental session but no information was given to participants regarding the predictive value of the color cues. This way, the effect of (cue) predictivity on subsequent target processing as such (without any confound related to cue directionality and explicit expectations about target location) could be explored thoroughly. Participants were simply instructed to attend either to the left or to the right side (relative to fixation) depending on the color of the cue, but were not given any information about the probability of target appearing in the cued location. Cueing was actually predictive for only half (either first or second depending on the specific subject's assignment) of the experiment (see below and participants were not informed about this change.

\section{Participants}

Twenty-four healthy adult participants (mean age 23.6 years, range 19-29 years, 8 males), with normal or corrected-to-normal vision freely took part in the experiment in exchange of thirteen euros compensation.

\section{Materials and procedure}

Stimuli were presented using E-Prime2 (Schneider, Eschmann, \& Zuccolotto, 2002) on a 17 inch screen positioned at a distance of $50 \mathrm{~cm}$ from the participant's head. A chin-rest was used to keep the head still and eye movements were continuously monitored using a Tobii T120 eye-tracker. Each trial started with a black fixation point (visual angle $0.5^{\circ}$ ), presented against a gray homogenous background (Figure 1). Trial sequence was triggered when the participant kept fixation (tolerance: $2^{\circ}$ ) for at least $250 \mathrm{~ms}$. Then, two placeholders (side $3.4^{\circ}$ ) were presented, one on the right and one on the left relative to the fixation point (distance from the centre $\approx 10^{\circ}$ ). After a constant 800 ms delay, the fixation point turned either yellow or blue. This colour change informed participants to orient either leftwards when yellow, or rightwards when blue (the mapping between 
color and direction was counterbalanced across participants). Either a short (160 ms) or a long SOA (600 ms) was randomly introduced between cue and target. The target stimulus consisted of a vertical or horizontal Gabor patch (contrast $100 \%$; subtending $\approx 3^{\circ}$ ) appearing in one of the two lateralized placeholders. Target duration was individually calibrated (see below). At target offset, the fixation point turned black again and the two placeholders were filled by a mask (checkerboard) until response or after 2.5 seconds elapsed. Half of the participants were required to press the "v" key for "vertical" (index finger left hand) and the " $n$ " key for "horizontal" (index finger right hand) while the other half received the opposite stimulus-response mapping. Instructions emphasized both speed and accuracy.

For sixteen practice trials the cued placeholder turned yellow/blue synchronously with the cue. This was done only during the practice to facilitate the learning of the arbitrary cue-location association. During the subsequent experimental session only the cue changed its color while the placeholder did not. There were two lists of experimental trials, one with cue predictivity set to $50 \%$ and another one with cue predictivity set to $90 \%$ [see Footnote 1]. List order was counterbalanced across participants. There were two short breaks, occurring after roughly $1 / 3$ and $2 / 3$ of the trials and dividing the experiment into three successive blocks of equal length/size. After 160 trials, in the middle of the second block, there was a change (not announced and not immediately noticeable) in the proportion of valid trials, from $50 \%$ to $90 \%$ or vice versa. Participants were not informed about the initial proportion of valid trials, nor about its sudden change during the experiment. After completing the experiment questionnaire adapted from Bartolomeo et al. (2006) and Risko and Stolz (2010) was administered (see the Supplementary Materials). It assessed whether participants had noticed a larger-than-chance association between color of the cues and target side, and/or any change in the strength of this relationship during the experiment. It also required estimating, separately for the two color cues (corresponding to leftward and rightward shift of attention), the percentage of targets appearing at the cued position (predictivity estimate).

We decided to present the questionnaire at the end of the experiment, instead of probing individual predictivity estimate online (during the experiment), in order not to bias individual awareness and to avoid providing hints about the change in predictivity. 
A

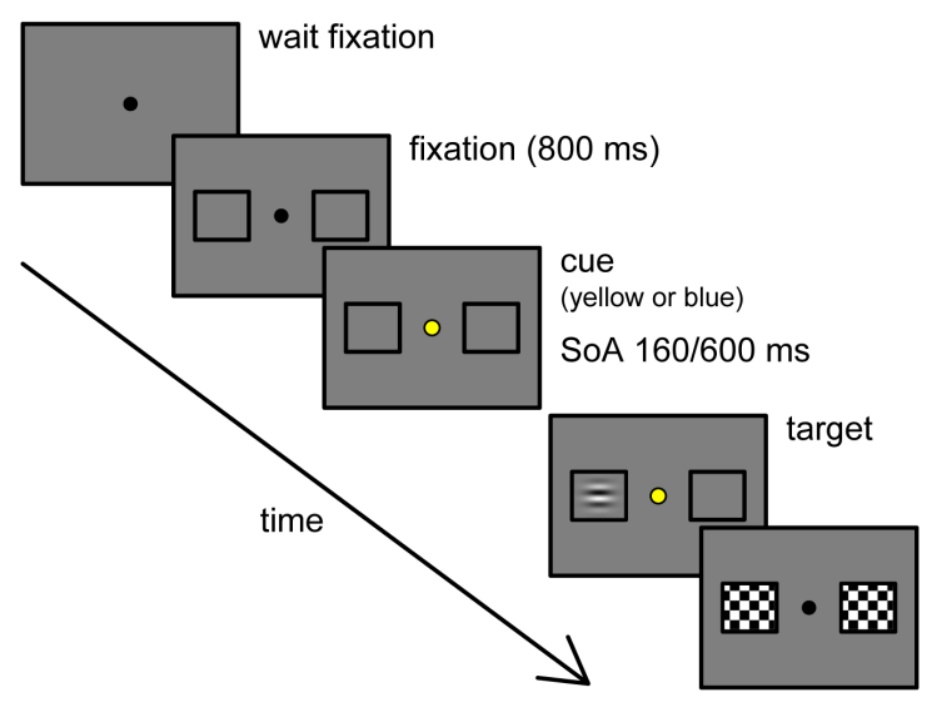

B

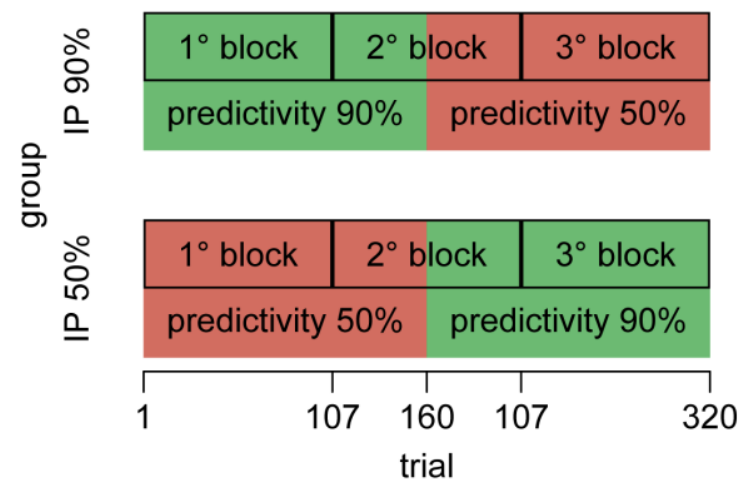

Fig. 1. (A) Trial structure. Color cueing was used to induce arbitrary cue-space associations. Half of the participants were instructed to orient attention to the right of fixation following a yellow cue and to the left after a blue cue. Half of the participants received the reverse assignment. The task was to discriminate the orientation (Horizontal vs. Vertical) of a unilateral Gabor patch by means of two response keys. Target duration was adjusted individually following a pre-experiment calibration procedure. In this representative trial a left target was presented after yellow cue. For the yellowleft/blue-right group this would have been a valid trial. For the yellow-right blue-left group this would have been an invalid trial. (B) Experimental design. There were two main trial lists, one with a cue predictivity of 50\% (upper right and lower left, in red) and the other one of $90 \%$ (upper left and lower right, in greed). For half of the participants (IP 50\%, lower panel) initial predictivity was set to $50 \%$ before changing to $90 \%$ after half of the trials, while for the other half of participants (IP $90 \%$, upper panel), the reverse order was used.

\section{Calibration procedure}

The aim of the calibration procedure ( 48 trials performed prior to experiment) was to adapt target duration to individual performance thereby avoiding floor/ceiling effects. Trial sequence was similar to experimental trials but there was no cue (the fixation point remained black). Initial target duration was set to $133 \mathrm{~ms}$ and then adjusted online (within the range 17-266 ms) through a weighted up-down staircase procedure (Kaernbach, 1991) with goal performance set to $75 \%$ accuracy. Duration values for the last 12 trials were averaged and used as individual target duration for the experiment. 


\section{Results}

\section{Reaction times}

Trials contaminated by eye movements $(1.60 \%)$ were excluded from further analyses. In these trials gaze position was detected (according to offline analysis) for more than 100ms consecutively outside of a circular area of $2^{\circ}$ of radius around the fixation point, in the interval between cue onset and the response. The data of two participants had to be excluded from statistical analyses, one due to excessive eye movements $(52.8 \%)$, and another one due to poor performance (error rate of $32 \%$, 2 SD above group average). RTs above $2500 \mathrm{~ms}(0.44 \%)$ were also discarded.

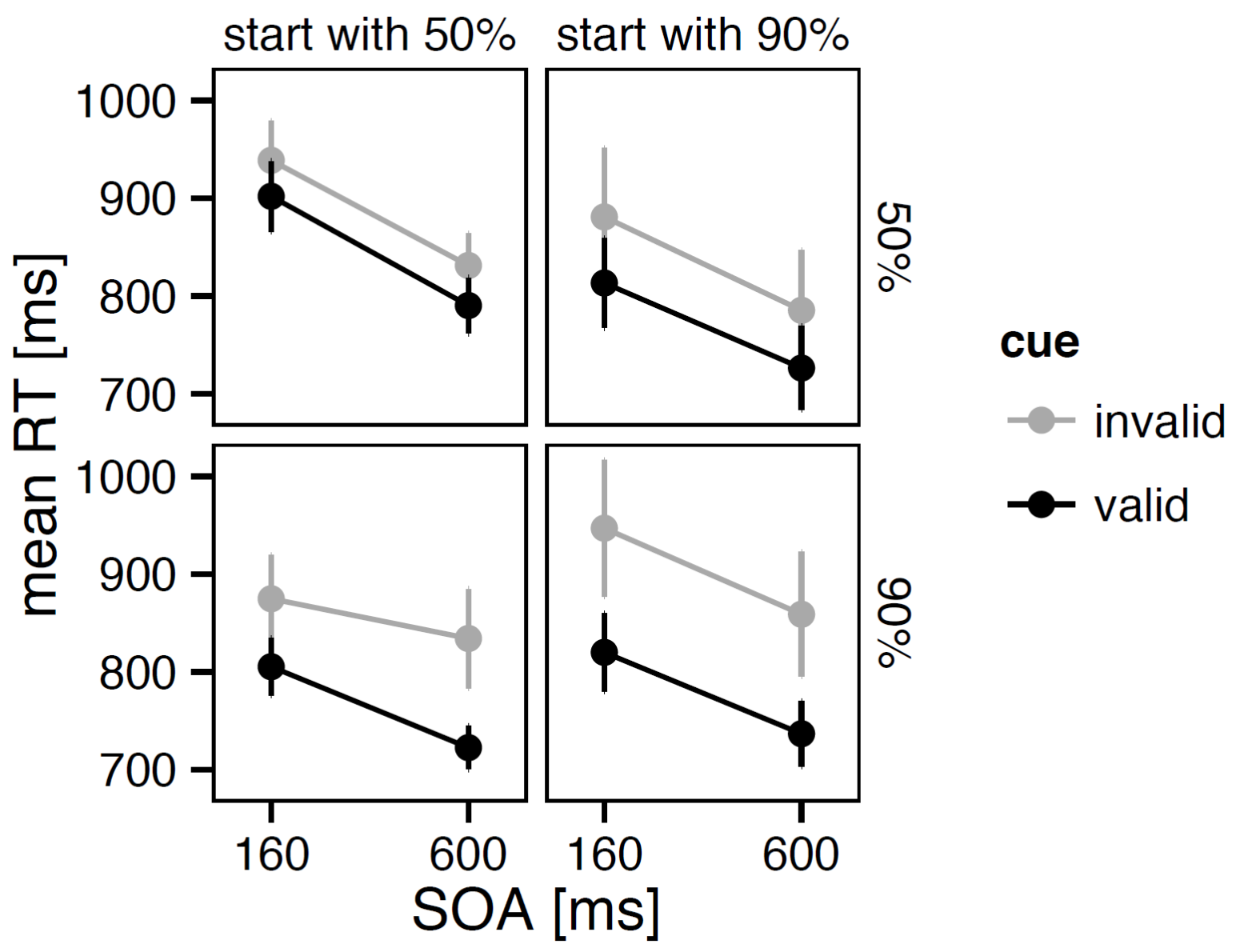

Fig. 2. Mean RTs (averaged across observers) for valid and invalid trials across the two SOAs (within each panel: $160 \mathrm{~ms}$ on the left and $600 \mathrm{~ms}$ on the right). The two panel rows correspond to the two levels of proportion valid and columns correspond to the two levels of initial predictivity IP. Upper left and lower right panels therefore show performance for the first block (for group IP50\% and IP90\%, respectively), while lower left and upper right panels show performance for the second block. Vertical error bars indicate bootstrapped SEM (Efron \& Tibshirani, 1986). 
Reaction times for correct responses were analyzed using a linear mixed-effects model (Pinheiro \& Bates, 2000), fitted with the open-source software R version 3.3.0 (R Core Team, 2015) and the lme4 library version 1.1-12 (Bates, Maechler, Bolker, \& Walker, 2014). To avoid potential issues related to the typical skewedness and non-normality of RT distributions, we implemented a Box-Cox transformation for RTs with $\lambda=-0.69$; a value which resulted from a maximum likelihood procedure (Box \& Cox, 1964).

We started with a model that had Trial validity (at the single trial level: valid vs. invalid), SOA (160 vs. $600 \mathrm{~ms}$ ), Proportion valid (at the block level: $50 \%$ vs. $90 \%$ of valid trials), and Block order assignment (at the experiment level: start with $50 \%$ vs. $90 \%$ of valid trials) and all possible interactions as fixed effects predictors; and subject as random effect predictor, or grouping factor. The model had random intercepts, thus allowed for different marginal RTs means for each participant, and also random slopes, for all the within-subjects factors (Trial validity, SOA and Proportion valid). We then proceeded by adding random slopes for each interaction between the within-subjects factors, except for the three-way interaction, because it caused a convergence failure of the optimization algorithm (i.e. its inclusion would have resulted in a model that was too complex to be properly supported by the data). However, none of these interaction factors improved the fit of the model (likelihood ratio test, all $p s>0.1$ ). Nevertheless, we included in the final model a random slope for the interaction between Proportion valid and Trial validity, as this interaction was the most interesting with respect to the main goal of the study. Additional analyses (not shown here) performed with models that included one or more of the random slopes for the remaining interaction terms resulted in a pattern of significant fixed-effects identical to that reported in Table 1.

To test fixed effects predictors, we performed a backward stepwise simplification: we iteratively removed a single term at a time, fitted the relative reduced model, and tested it against the full model. At each step of the process we tested all possible models resulting from the exclusion of a single term (maintaining marginality). Next we refitted the model excluding all the terms that could be dropped without a significant worsening of the model fit (likelihood-ratio tests, $\alpha=.05)$. The process was repeated until no more terms could be dropped without leading to a significant decrease in the likelihood of the model or a violation of marginality (on the importance of marginality in regression analysis, we refer the reader to Nelder, 1977; Venables, 1998). For each step of the simplification process we listed the regression terms that could be dropped (Table 1), along with the results of the corresponding likelihood ratio tests. The estimates of fixed effects and variance/covariance components for the linear mixed-effects models used in the analysis are reported in Supplementary Materials Table S1. 
Crucially for our purposes, the significant interaction between Trial validity (at trial level) and Proportion valid (at block level) showed that the validity effect, here unbiased because triggered without directional cues, was context-dependent because modulated by the proportion of valid trials (Table 1). The model predicted faster RTs in valid as compared to invalid trials, and this was confirmed by means of paired, two-tailed, t-tests in both blocks separately [block 50\%, mean difference 51ms, $t(21)=3.90, p=0.0008$; block 90\%, mean difference 99ms, $t(21)=4.04, p=$ 0.0006]. The effect in the non-predictive block was still significant when the considering only the IP50 participants, that is when the non-predictive block was performed first and any "contamination" from the predictive block could be excluded, $t(11)=2.41, p<0.05$.

We also found a significant main effect of SOA, indicating faster RTs at the 600ms delay (see Figure 2 and Table 1), and a significant interaction between Proportion valid and Block order assignment. Presumably, this interaction indexed a learning effect, whereby participants were slower in the first block than in the second block depending on the initial (and unknown) cuepredictivity assignment, suggesting an impact of trial history on general response speed. Moreover, the main effect of SOA is probably a direct consequence of the longer time period available for preparing a manual response to the target in the $600 \mathrm{~ms}$ compared to the $160 \mathrm{~ms}$ SOA condition. This effect is not uncommon (e.g. Fuchs \& Ansorge, 2012; Fuchs, Ansorge, Huber-Huber, Höflich, Lanzenberger, 2015). Of note, SOA did not interact with validity. 
Table 1

Backward stepwise simplification of LMM model

(Box-Cox transformed RTs with $\lambda=-0.69$ )

\begin{tabular}{l|l|c|c|c}
\hline Step & \multicolumn{1}{|c|}{ Regression term } & Df & $\chi^{\mathbf{2}}$ & $\boldsymbol{p}$ \\
\hline 1 & Trial validity * SoA * Proportion valid * Block order & 1 & 0.05 & 0.82 \\
\hline 2 & Trial validity * SoA * Proportion valid & 1 & 0.14 & 0.71 \\
& Trial validity * SoA * Block order assignment & 1 & 0.13 & 0.72 \\
& Trial validity * Proportion valid * Block order & 1 & 0.07 & 0.79 \\
& SoA * Proportion valid * Block order assignment & 1 & $<0.01$ & 0.99 \\
\hline 3 & Trial validity * SoA & 1 & 1.45 & 0.23 \\
& SoA * Proportion valid & 1 & 2.02 & 0.15 \\
& SoA * Block order assignment & 1 & 0.27 & 0.60 \\
& Trial validity * Block order assignment & 1 & 0.12 & 0.72 \\
\hline $4($ end $)$ & Trial validity * Proportion valid & 1 & 8.02 & $<0.001$ \\
& Proportion valid * Block order assignment & 1 & 14.92 & $<0.001$ \\
& SoA & 1 & 38.67 & $<0.001$ \\
\hline Wald & Trial validity & 1 & 20.76 & $<0.001$ \\
& Proportion valid & 1 & 4.57 & 0.03 \\
& Block order assignment & 0.06 & 0.80
\end{tabular}

Table 1. Backward stepwise simplification of the linear mixed effects model used in the analysis of RTs. Note that in the final step $\left(4^{\text {th }}\right)$ the likelihood ratio tests were significant: these factors could not be excluded without a significant worsening of the model fit (as indexed by a significant drop in the likelihood of the model). For completeness, in the last row we report Wald type II tests for the regression terms that could not be tested, during the stepwise simplification, without violating marginality (i.e. because these terms were included in significant higher order interactions).

Next, we assessed whether the subject-specific coefficients (that is the conditional modes of the random effects) coding for the cue validity effect (difference in RTs between valid and invalid trials) differed according to the individual awareness of the relation between cue color and target position. Participants were divided into two groups according to their subjective awareness of the cue-target relationship, on the basis of the response provided to question number 3 "Have you perceived any contingency between the colour of the central dot and the position of appearance of the target?". Those who reported having noticed a systematic association between the cue and the target location entered the aware group $(\mathrm{n}=11)$. Those who failed to notice such an association entered the non-aware group $(n=11)$. No significant group difference in the size of the cue validity effect was found $[t(20)=0.64, p=0.53]$. We also examined whether adding subjective awareness (aware vs non-aware) as a predictor could improve the fit of the final linear mixed-effects model used in RTs analysis (Table 1). When we added subjective awareness as fixed-effect predictor, 
including interactions with all other predictors, no improvement in the goodness of fit was observed (likelihood ratio tests, all $p>0.1)$. Similarly, we assessed whether those participants $(\mathrm{n}=11)$ who positively responded to question 5 "Do you have the feeling that cue-target relation changed during the experiment ?" (awareness of predictivity change) had a significantly larger speed difference in validity between the two types of block (90\% and 50\%) by looking at the corresponding subjectspecific coefficients coding for the interaction between Trial validity and Proportion valid. However, no significant group difference emerged $[t(20)=0.17, p=0.87]$. Further, adding the awareness of predictivity change as predictor in the mixed-effects model, including all possible interactions, did not improve the goodness of fit of the model either (likelihood ratio tests, all $p>0.1)$. These findings allowed us to rule out, at least partly, the possibility that different validity effects were mostly due to participants adjusting their efforts/strategies according to their degree of insight/awareness of the cue-target contingencies.

\section{[Footnote 1]}

Finally, we performed a more fine-grained analysis to test whether fluctuations in the size of validity effects across the experiment could also be predicted at a given time point according to the immediately preceding and most recent trials. We reasoned that, in case the amplitude of the validity effect is determined, as we contend, by cue-target contingencies, it should be possible to account for the fluctuations in its size by taking into account the recent trial history. To this aim, we took advantage of the fact that mixed-effects models do not require averaging of data within each cell in the experimental design, and thus allow testing predictors that vary on a trial-by-trial basis. For each trial, we counted the number of immediately preceding, consecutive valid trials (i.e., not interrupted by an invalid trial), and added it as a predictor, in interaction with Trial validity, in the final model resulting from the previous simplification procedure. In other words this predictor consisted in the number of valid trials between the current trial and the last invalid trial, and therefore it was set to zero for each trial that immediately followed an invalid one. The rationale of this analysis is the following: if adding this predictor on top of the interaction between Trial validity and proportion Valid increases significantly the goodness of fit, this indicates that there are systematic fluctuations in the validity effect that depend on the recent trial history and which cannot be accounted for exclusively by the block-level predictor Proportion valid. In line with this assumption, we found that the inclusion of this predictor in the model significantly increased the goodness of fit, $\chi^{2}(1)=15.74, p<0.001$. This finding is valuable because it suggests that at a given, arbitrary timepoint, the (predicted) validity effect (difference between the expected RT in case of invalid cue minus the expected RT in case of valid cue) was reliably modulated by the cue-location coupling experienced by the subjects during the previous trials, above and beyond the effect of 
Proportion valid at the block level. More specifically, this result suggests that framing the effect as a function of previous trial history allows to track rapid fluctuations in the cueing effects that are neglected by the global model. However, as a word of caution, it is important to point out that the predictivity at the block level and at the single trial level could be only partly orthogonalized in our paradigm.

Response accuracy

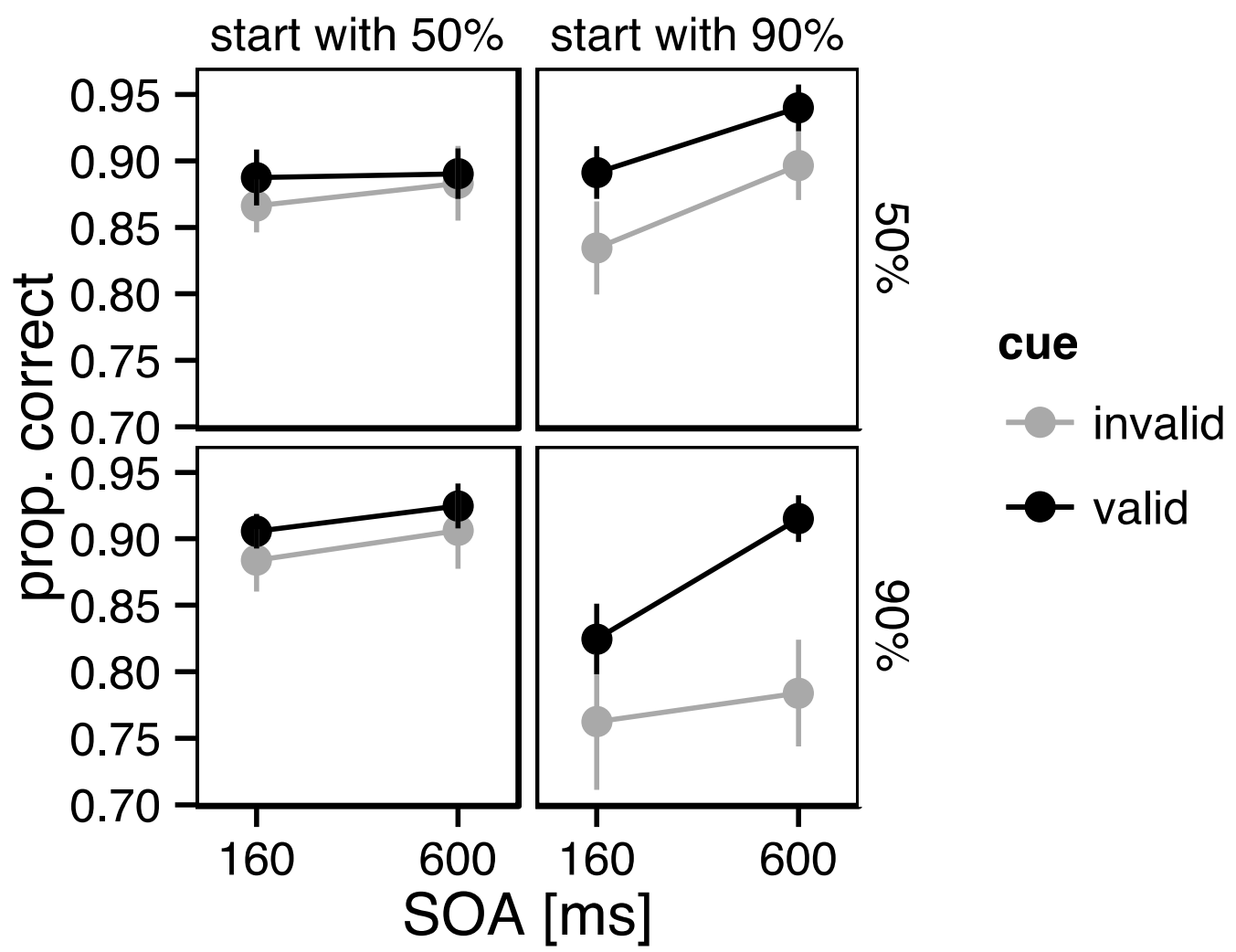

Fig. 3. Average proportion of correct responses, separately for each level of validity, cue predictivity (upper vs lower panels), order (left vs right panels) and SOA (columns within panels). Vertical bars indicate bootstrapped SE (Efron \& Tibshirani, 1986). 
Table 2

Backward stepwise simplification of GLMM model

\begin{tabular}{|c|c|c|c|c|}
\hline Step & Regression term & df & $\chi^{2}$ & $p$ \\
\hline 1 & $\begin{array}{l}\text { Trial validity } * \text { SOA } * \text { Proportion valid } * \text { Block order } \\
\text { assignment }\end{array}$ & 1 & 0.41 & 0.52 \\
\hline \multirow[t]{4}{*}{2} & Trial validity $*$ SOA $*$ Proportion valid & 1 & 1.09 & 0.30 \\
\hline & Trial validity $*$ SOA $*$ Block order assignment & 1 & 1.29 & 0.27 \\
\hline & Trial validity $*$ Proportion valid $*$ Block order assignment & 1 & 0.04 & 0.83 \\
\hline & SOA $*$ Proportion valid $*$ Block order assignment & 1 & 0.26 & 0.61 \\
\hline \multirow[t]{6}{*}{$3($ end $)$} & Trial validity $*$ SOA & 1 & 0.12 & 0.72 \\
\hline & Trial validity $*$ Proportion valid & 1 & 0.45 & 0.50 \\
\hline & SOA * Proportion valid & 1 & 0.18 & 0.67 \\
\hline & Trial validity $*$ Block order assignment & 1 & 4.17 & 0.04 \\
\hline & SOA * Block order assignment & 1 & 4.65 & 0.03 \\
\hline & Proportion valid $*$ Block order assignment & 1 & 16.87 & $<0.0001$ \\
\hline \multirow{4}{*}{$\begin{array}{l}\text { (Wald } \\
\text { tests) }\end{array}$} & SOA & 1 & 16.36 & $<0.0001$ \\
\hline & Trial validity & 1 & 16.93 & $<0.0001$ \\
\hline & Proportion valid & 1 & 1.73 & 0.18 \\
\hline & Block order assignment & 1 & 0.92 & 0.16 \\
\hline
\end{tabular}

Table 2. Backward stepwise simplification of the generalized linear mixed effects model used in the analysis of response accuracy. In the final row, we report Wald tests for the remaining factors.

Before analyzing response accuracies, we ensured that our calibration procedure returned an appropriate individual target duration value by computing the correlation over subjects between the individual value of target duration and the overall proportion of correct responses. No evidence for a significant correlation was found $[r(20)=0.14, p=0.53]$, indicating that the task was equally challenging for all participants included in the analysis.

Also response accuracy was analyzed with a generalized linear mixed effects model (GLMM), now with the logit as link function (see Figure 3 for averaged data). The model had subject as grouping factor, and included random slopes for the same terms as the model used to analyze RTs. Adding additional random slopes did not improve the fit of the model (likelihood ratio test, all $p>0.1$ ), nor changed the pattern of significant fixed-effects (not shown here). The estimates of fixed effects and variance/covariance components for the linear mixed-effects models used in the analysis are reported in Supplementary Materials Table S2.

As opposed to RTs, no modulation of validity emerged in the non-predictive block when it was the first presented [IP 50 participants], $t(11)=0.64, p=0.53$. 
Finally, we performed a one-way ANOVA on accuracy differences between validly and invalidly cued trials. We were interested in investigating whether cueing effects differed among participants according the individually-reported awareness of any systematic relation between the color of the cue and side of appearance of the target. This analysis did not reveal any difference $[F(1,20)=1.85, p=0.19]$.

Even if we found no evidence for a systematic modulation of the proportion valid on response accuracy by the trial context (proportion valid), we investigated whether there were some fluctuations in the size of the validity effect that could be explained by taking into account the recent trial history. Following the same rationale as in the analysis of the RTs above, we added as a predictor (in interaction with Trial validity) the number of immediately preceding consecutive valid trials, computed individually for each trial. In contrast with the analysis of RTs, the addition of this predictor did not improve the fit of the model, $\chi^{2}(1)=0.66, p=0.47$. In sum, we found no evidence for a modulation of response accuracy by the recent trial history, unlike the RTs.

We also investigated whether the responses to the (post-experiment) questionnaire could influence task performance when titrated using response accuracy, by including additional predictors in the final GLMM model. First we added subjective awareness (aware vs. non-aware) of the cue-target relationship, but failed to observe any variation across participants in the size of the validity effect that could be explained by this predictor (likelihood ratio tests, all $p s>0.1$ ). Similarly, including the awareness of predictivity change (in interaction with Proportion valid and Trial validity) as predictor in the model, did not improve the fit of the model either (likelihood ratio tests, all $p s>0.1)$.

\section{Awareness of cue predictivity and influence on cueing effect}

To examine whether performance was influenced, at individual level, by the perceived proportion of valid trials, we compared post-experiment ratings with the amplitude of the validity effect resulting from the computer-based task. For this purpose, we calculated exploratory correlations between the individual cueing effects (for either RTs or accuracy) and the individual cue predictivity estimate (see Risko \& Stolz, 2010a for a similar procedure). We computed these correlations either separately for the two cue colors and SOA, as well as averaging individual estimates and measured effects over color and SOA, respectively. These tests did not reveal any significant correlation between the two variables: the correlation values ranged from -0.12 to 0.32 , and the $p$ values ranged from 0.16 to 0.89 . However, since our sample size is modest, caution is 
needed when interpreting this non-significant result (the absence of evidence does not prove the evidence of absence). It is also possible that the detection of a significant correlation is made less likely due to the noisy nature of differences scores (cf. Cronbach \& Furby, 1970).

\section{Discussion}

In this study, we instructed participants to orient attention according to the colour of a central nondirectional cue that, unbeknown to them, was predictive of target position (90\% valid) in half of the experiment and non-predictive (50\% valid) in the other half. The use of non directional cues was a distinctive task characteristic and allowed to avoid unwarranted orienting effects due to cue asymmetries. Our results showed that a faster discrimination of targets occurred when their location matched the one indexed by the colour, compared to the situation where they appeared at the invalid location. This cueing effect was already present in the non-predictive block but was larger in the predictive one, and its amplitude (when considering RT speed) resulted to be modulated the recent trials history (i.e. by the number of preceding consecutive valid trials). The strength of the cueing effect on RTs therefore appears to be strongly modulated by the learning of cue/colortarget/location contingencies. In this specific case contingencies were arbitrary and therefore not apriori biased as opposed, for instance, to the use of asymmetrical cues like arrow cues (Lambert et al., 2006). Moreover, awareness of these contingencies (as indicated by post-experiment ratings) did not modulate the strength of the spatial orienting effect. The effect of the proportion of valid trials and its interaction with trial context were roughly constant across the two different SOAs used, allowing to exclude the more effective engagement of a slow, voluntary, component over less effective engagement of fast and automatic - possibly associative- processes.

Our results suggest that the proportion valid effect on RTs is the outcome of an automatic process, which modulates attention orienting on the basis of (rapidly learned or instructed) spatial-temporal contingencies in the environment (Zhao, Al-Aidroos, \& Turk-Browne, 2013), possibly through a mechanism similar to repetition priming (Dobbins, 2004; Logan, 1990) and can act outside awareness and without explicit strategies.

We also found that the proportion valid effect on accuracy was modulated by the more general context, at experiment level (block order assignment), as opposed to the single trial level in the case of RTs. Specifically, we observed that the extent of cueing effects on response accuracy depended only on which block (90\% or $50 \%$ predictivity) was presented first. In contrast, cueing effect on response accuracy was not modulated by changes in predictivity during the experiment. Given that response accuracy is thought to directly reflect the quality of target representation, it seems natural 
to assume that it should be more strongly linked to overt cognitive strategies. Therefore, we suggest that cueing effects on response accuracy might largely depend on the task set adopted, which seems to be fully configured during the initial part of the experiment (where specific expectations are created based on the task instructions provided and cue predictivity initially encountered). This interpretation implies that slightly different task sets can be determined by different level of predictivity during the initial phase of the experiment, that can then linger regardless of changes in predictivity encountered during the experiment.

Our interpretation of the different contingencies effects on RTs/accuracy is in line with the influential proposal by Prinzmetal, McCool, and Park, (2005) according to which automatic allocation of spatial attention would not affect the underlying representations and would show its signature on RTs only. We point out however that, in the more commonly used detection tasks, this dissociation cannot emerge due to the almost flawless performance usually exhibited (by healthy participants).

The present results cast doubts on the almost-never-tested assumption that predictive cueing leads to "pure" voluntary orienting of spatial attention. Rather, it seems more appropriate to conclude that several earlier studies that attributed shifts of spatial attention to a voluntary control mechanism could actually be, at least in part, explained by the effects of target predictability or cue predictivity per se, in the absence of voluntary control (Prinzmetal, McCool \& Park, 2005). As a matter of fact, predictive cueing is still considered the gold standard in research on spatial orienting of attention (Corbetta \& Shulman, 2011). The use/non-use of predictive cueing is believed to distinguish endogenous from exogenous mechanisms (Awh, Belopolsky \& Theeuwes, 2012). The present results suggest that such a dissociation may actually be conflated by differences in levels of cue predictivity, rather than on core attentional components (see Doricchi et al., 2010, for a similar view). While our new results clearly confirm the efficacy and sensitivity of the Posner paradigm to disentangle the contribution of several key processes underlying visuo-spatial attention in humans, at the same time they also show that predictive cueing does not only induce pure voluntary orienting. In this context, our novel behavioral findings are also compatible with the assumption that expectations and predictions are two major determinants of the efficacy of spatial orienting (for conceptually similar findings see for instance Melloni, Schwiedrzik, Müller, Rodriguez \& Singer, 2011). At the same time, however they can hardly subsume the standard dichotomy between endogenous and exogenous attention effects, rather suggesting that these spatial orienting effects always necessarily result from the integration of sensory signals with top-down input (Macaluso \& Doricchi, 2013). The multifaceted picture provided by RTs and accuracy unambiguously calls for a very strong modulation of performance caused by cue-target contingencies. 
Using different approaches or experimental set-ups than the one used here, some early studies in the field already emphasized the importance of the "proportion valid" trials (or cue predictivity) to account for spatial orienting effects (Eriksen \& Yeh, 1985; Johnson \& Yantis, 1995). Puzzlingly enough, these initial hints did not lead to a systematic investigation of the possibly crucial role of predictivity in shaping spatial orienting effects. Accordingly, a specific contribution of our study is to show, using a novel methodology and arbitrary cue/target associations, that shifts of spatial attention are strongly influenced by predictivity levels while robust orienting could, at the same time, be reliably triggered by (the use of) simple instructions.

A significant validity effect for RTs was found also for the non-predictive block. An open issue is therefore to what extent these cue-target (spatial) associations leading to changes in response speed are involuntary or whether, alternatively, genuine voluntary mechanisms or components are also involved. In a seminal study, Lambert, Naikar, McLachlan, and Aitken, (1999) reported an implicit cueing effect on RTs (peripheral symbolic cues and peripheral targets) unrelated to participants' awareness of the cue-target relationship and perceptual awareness of the cue itself, corroborating the notion that spatial shifts of attention could be triggered by implicit cue-target associations. However, the results of Bartolomeo et al. (2007) seem to suggest that some room for purely voluntary components exists. They manipulated the proportion of valid trials in a cued detection task (central arrow or peripheral cue). A larger orienting effect following central cueing was observed for participants who, according to a questionnaire, were aware of the predictivity levels. The link between awareness of predictivity and cueing effects led Bartolomeo and colleagues to conclude that spatial cueing effects may be due to a voluntary component, because spatial orienting more reliably occurred when cue predictivity level accessed awareness.

Risko and Stolz (2010a) proposed implicit learning as an alternative explanation for the proportion valid effect. They manipulated the predictivity (e.g. 50\% vs. $75 \%$ ) of either a peripheral or a central arrow cue. In both conditions cueing effects increased with increase in predictivity. A questionnaire showed that following peripheral but not central cueing participants correctly identified the two different levels of predictivity used. Crucially (and similarly to the present results), the estimated levels of predictivity were unrelated to the actual cueing effects on both RTs and accuracy. Given the robust cueing effect obtained despite the short SOA $(150 \mathrm{~ms})$, these authors suggested that endogenous orienting is (considered) too slow to modulate the proportion valid effect (which was present irrespective of participants' awareness of cue predictivity). They concluded that the proportion valid effect results from involuntary attentional capture and implicit learning (see also Peterson \& Gibson, 2011). Chica \& Bartolomeo (2010) argued that, although implicit learning might play a role in this phenomenon, the concurrent involvement of strategic control cannot be 
excluded (see also Risko \& Stolz, 2010b). Earlier findings (Risko, Blais, Stolz, \& Besner, 2008a,b) suggest a modulation of spatial orienting performance due to frequency differences, whereby the more frequent condition would give rise to a more efficient processing than the less frequent condition (see Cutini et al., 2008 for converging evidence in task-switching).

A potential lack of systematic association between the awareness of cue predictivity and the observed cueing effects would suggest that spatial orienting based on predictive cues cannot be qualified as purely "voluntary” (Peterson \& Gibson 2011; Risko et al., 2008; Risko \& Stolz, 2010a). The non-significant correlations we found are subject to caution and deemed preliminary only. At any rate, the potential absence of an association between awareness of cue predictivity and amplitude of the cueing effects (even at long SOAs), if confirmed by future studies, would reinforce the notion that in predictive cueing the voluntary component is not necessarily the most important determinant of performance.

More generally, the present findings extend the notion that cue-target associations can strongly influence performance (López-Ramón, Chica, Bartolomeo \& Lupiáñez, 2011) even when cues are devoid of directionality. This association has been recently proven so strong to counteract the effect of hemispatial neglect (as highlighted in computer based tasks, see Bonato, 2012) in the contralesional hemispace (Wansard, Bartolomeo, Vanderaspoilden, Geurten, \& Meulemans, 2015). Our study goes beyond this intriguing observation by showing that implicit learning-like effects hold not only for the location where the cue is presented, as is the case with peripheral cues (Wansard et al., 2015), but can also be evidenced at a higher level. In our task, spatial properties were not immediately decoded or available, but the information on cue-target probability was nonetheless timely extracted from the arbitrary color feature at fixation to subsequently enhance the (visual) processing of a non-spatially overlapping target.

Our new findings extend the results reported in a recent series of studies by Vossel and collaborators, which focused on RTs modulation resulting from changes in cue-target predictability. In these studies, cues were mostly directional and participants were told to expect changes in cue target occurrence through the task. In a first study, it was demonstrated that an explicit arrow direction can trigger saccadic movements more efficiently when predictive of target position (Vossel, Mathys et al., 2014), and its effect is influenced by cholinergic stimulation (Vossel, Bauer et al., 2014). Applying the same logic to a search task revealed an increase in the cueing effect on RTs the more the cue was abstract (Dombert, Fink \& Vossel, 2016). The effects related to the proportion of valid trials were limited when the cue was painted in the color to be looked for (e.g. "red") and more prominent when it suggested the first two syllables (e.g. "re"). 
Noteworthy, in domains other than spatial orienting/attention, the role of contextual effects and associative/implicit learning are becoming more and more conceived, albeit not always explicitly, as major determinants of performance (for a recent review, see Abrahamse, Braem, Notebaert, \& Verguts, 2016; see also Braem, Hickey, Duthoo, \& Notebaert, 2014; Duthoo, Abrahamse, Braem, Boehler, \& Notebaert, 2014; Schmidt, 2013; Rieth \& Huber, 2013).

To conclude, the present results show that a change, unknown to the participants, in the reliability the cue had in predicting target location (proportion of valid trials) deeply affected the spatial orienting of attention (to the target location) rather independently from participant's awareness of cue predictivity. With respect to RT effects: (i) the size of spatial cueing effects is strongly modulated by cue predictivity; (ii) immediately after cue presentation and persisting at a longer cuetarget SOA, and (iii) fluctuations in the size of the cueing effects can be predicted by considering the recent trial history. In accuracy, these spatial cueing effects were strongly modulated by block history, and were reliable only when the participants experienced as first a strong/clear cue target association.

For more than 40 years and since the groundbreaking work of Michael Posner, researchers have striven to isolate the specific contribution(s) of voluntary mechanisms responsible for the spatial orienting of attention, using clever experimental designs. However, often, these designs entail that spatial orienting repeats over again and again across several hundred (sometimes thousands) of trials, and can therefore be best captured by computing mean scores. This notion seems, in any case, a priori questionable because initial volition can rapidly turn into automatic processing. Crucially, here we showed that, different levels of cue predictivity at the beginning of the experiment induce different task set configurations (accuracy) and implicitly shape the size and strength of cueing effects in the short run, as evidenced by a systematic influence of the recent trial history (RTs). These effects seem to occur relatively independently from participants' awareness. As such, our new results emphasize that the underlying orienting processes activated by predictive cueing are probably far more complex and dynamic than initially thought, and they do not correspond to a simple instruction-based voluntary mechanism.

\section{[Footnote 1]}

Of note, the two levels of cue predictivity used here are only indicative because "real" cue predictivity changes on trial-by-trial basis. 


\section{Compliance with Ethical Standards:}

Funding: MB was funded by a FWO Pegasus/Marie Curie IEF Fellowship within the $7^{\text {th }}$ framework program (Project 625378 "SpaceLOAD").

Conflict of Interest: MB declares that he has no conflict of interest. ML declares that he has no conflict of interest. SP declares that she has no conflict of interest. GP declares that he has no conflict of interest.

Ethical approval: All procedures performed in studies involving human participants were in accordance with the ethical standards of the institutional research committee and with the 1964 Helsinki declaration and its later amendments or comparable ethical standards.

Informed consent: Informed consent was obtained from all individual participants included in the study.

\section{References}

Abrahamse, E., Braem, S., Notebaert, W., \& Verguts, T. (online first, 2016). Grounding Cognitive Control in Associative Learning. Psychological Bulletin.

Akaike, H. (1974). A new look at the statistical model identification. IEEE Transactions Automatic Control, 19, 716-723.

Awh, E., Belopolsky, A.V., \& Theeuwes, J. (2012). Top-down versus bottom-up attentional control: a failed theoretical dichotomy. Trends in Cognitive Sciences, 16, 437-443.

Baayen, R.H. (2005). Data mining at the intersection of psychology and linguistics. In A. Cutler (ed), Twenty-First Century Psycholinguistics: Four Cornerstones (pp. 69-83). Mahwah, NJ:

Erlbaum. 
Box, G.E.P. \& Cox, D. R. (1964) An analysis of transformations. Journal of the Royal Statisistical Society, Series B. 26, 211-246.

Bartolomeo, P., Decaix, C., \& Siéroff, E. (2007). The phenomenology of endogenous orienting. Consciousness and Cognition, 16, 144-161.

Bates, D.M., \& Sarkar, D. (2007). lme4: Linear mixed-effects models using S4 classes, R package version 0.99875-6.

Bates, D., Maechler, M., Bolker, B., \& Walker, S. (2014). lme4: Linear mixed-effects models using Eigen and S4. R package version 1.1-7. Retrieved from http://cran.r-project.org/package=lme4

Bonato, M., Priftis, K., Marenzi, R., \& Zorzi, M. (2009). Normal and impaired reflexive orienting of attention after central nonpredictive cues. Journal of Cognitive Neuroscience, 21, 745-759.

Bonato, M. (2012). Neglect and extinction depend greatly on task demands: A review. Frontiers in Human Neuroscience 6:195.

Bonato, M. (2015). Unveiling residual, spontaneous recovery from subtle hemispatial neglect three years after stroke. Frontiers in Human Neuroscience 9:413.

Box, G. E. P., \& Cox, R. (1964). An Analysis of Transformations. Journal of the Royal Statistical Society. Series B (Methodological), 26(2), 211-252.

Braem, S., Hickey, C., Duthoo, W., \& Notebaert, W. (2014). Reward determines the contextsensitivity of cognitive control. Journal of Experimental Psychology: Human Perception \& Performance, 40,1769-1778. doi: 10.1037/a0037554.

Burnham, K. P., \& Anderson, D. R. (2002). Model Selection and Multimodel Inference: A Practical Information-Theoretic Approach. New York, US: Springer-Verlag.

Carrasco, M. (2011). Visual Attention: The past 25 years. Vision Research, 51, 1484-1525.

Chica, A.B., \& Bartolomeo, P. (2010). Unconscious strategies? Commentary on Risko and Stolz (2010): The proportion valid effect in covert orienting: Strategic control or implicit learning? Consciousness and Cognition, 91, 443-444. 
Chica, A.B., Martín-Arévalo, E., Botta, F., \& Lupiáñez, J. (2014). The Spatial Orienting paradigm: how to design and interpret spatial attention experiments. Neuroscience \& Biobehavioural Reviews. 40:35-51. doi: 10.1016/j.neubiorev.2014.01.002.

Corbetta, M., \& Shulman, G. L. (2011). Spatial neglect and attention networks. Annual Review of Neuroscience, 34, 569-599.

Cronbach, L.J., \& Furby, L. (1970). How we should measure "change": Or should we? Psychological Bulletin, 74, 68-80.

Cutini, S., Scatturin, P., Menon, E., Bisiacchi, P.S., Gamberini, L., Zorzi, M., \& Dell’Acqua, R. (2008). Selective activation of the superior frontal gyrus in task-switching: an event-related fNIRS study. NeuroImage, 42, 945-955.

Dobbins, I.G. (2004). Cortical activity reductions during repetition priming can result from rapid response learning. Nature, 428, 316-319.

Dombert, P.L., Fink, G.R., \& Vossel, S. (2016) The impact of probabilistic feature cueing depends on the level of cue abstraction. Experimental Brain Research, 234, 685-694.

Doricchi, F., Macci, E., Silvetti, M., \& Macaluso, E. (2010). Neural correlates of the spatial and expectancy components of endogenous and stimulus-driven orienting of attention in the posner task. Cerebral Cortex, 20, 1574-1585.

Duthoo, W., Abrahamse, E.L., Braem, S., Boehler, C.N., \& Notebaert W. (2014). The heterogeneous world of congruency sequence effects: an update. Frontiers in Psychology 5:1001. Eimer, M. (1997). Uninformative symbolic cues may bias visual-spatial attention: behavioral and electrophysiological evidence. Biological Psychology, 46, 67-71.

Efron, B., \& Tibshirani, R. (1986). Bootstrap Methods for Standard Errors, Confidence Intervals, and Other Measures of Statistical Accuracy. Statistical Science, 1(1), 54-75. Retrieved from http://www.jstor.org/stable/2245500

Eriksen, C.W., \& Yeh, Y.Y. (1985). Allocation of attention in the visual field. Journal of Experimental Psychology: Human Perception \& Performance, 11, 583-587. 
Fuchs, I., \& Ansorge, U., (2012). Unconscious cueing via the superior colliculi: Evidence from searching for onset and color targets. Brain Sciences, 2, 33-60.

Fuchs, I., Ansorge, U., Huber-Huber, C., Höflich, A., \& Lanzenberger, R. (2015). S-ketamine influences strategic allocation of attention but not exogenous capture of attention. Consciousness and Cognition, 35, 282-294

Hommel, B., Pratt, J., Colzato, L., \& Godijn, R. (2001). Symbolic control of visual attention. Psychological Science, 12, 360-365.

Johnson, D.N., \& Yantis, S. (1995). Allocating visual attention: Tests of a two-process model. Journal of Experimental Psychology: Human Perception \& Performance, 21, 1376-1390.

Jonides, J. (1981). Voluntary versus automatic control over the mind's eye's movement. In J. B. Long \& A. D. Baddeley (Eds.), Attention and performance IX (pp. 187-203). Hillsdale, NJ: Erlbaum.

Kaernbach, C. (1991). Simple adaptive testing with the weighted up-down method. Perception \& Psychophysics, 49(3), 227-229.

Kinoshita, S., Mozer, M.C., Forster, K.I. (2011). Dynamic adaptation to history of trial difficulty explains the effect of congruency proportion on masked priming. Journal of Experimental Psychology: General, 140(4), 622-636.

Koshino, H., Warner, C.B., \& Juola, J.F. (1992). Relative effectiveness of central, peripheral, and abrupt-onset cues in visual attention. Quarterly Journal of Experimental Psychology, 45(4), 609631.

Lambert, A., Naikar, N., McLachlan, K., \& Aitken, V. (1999). A new component of visual orienting: Implicit effects of peripheral information and subthreshold cues on covert attention. Journal of Experimental Psychology: Human Perception and Performance, 25, 321-340.

Lambert, A., Roser, M., Wells, I., \& Heffer, C. (2006). The spatial correspondence hypothesis and orienting in response to central and peripheral spatial cues. Visual Cognition, 13(1), 65-88. 
López-Ramón, M.F., Chica, A.B., Bartolomeo, P., \& Lupiáñez, J. (2011). Attentional orienting and awareness: Evidence from a discrimination task. Consciousness and Cognition, 20, 745-755. Logan, G.D. (1990). Repetition priming and automaticity: Common underlying mechanisms? Cognitive Psychology, 22, 1-35.

Losier, B.J., and Klein, R.M. (2001). A review of the evidence for a disengage deficit following parietal lobe damage. Neuroscience Biobehavioral Reviews, 25, 1-13.

Macaluso, E., \& Doricchi, F. (2013). Attention and predictions: control of spatial attention beyond the endogenous-exogenous dichotomy. Frontiers in Human Neuroscience. 7:685. doi: 10.3389/fnhum.2013.00685.

Melloni, L., Schwiedrzik, C.M., Müller, N., Rodriguez, E., \& Singer, W. (2011). Expectations change the signatures and timing of electrophysiological correlates of perceptual awareness.

Journal of Neuroscience, 26;31(4),1386-1396.

Nelder, J.A. (1977). A Reformulation of Linear Models. Journal of the Royal Statistical Society. Series A (General), 140, 48-77. doi:10.2307/2344517

Olk, B., Hildebrandt, H., \& Kingstone, A. (2010). Involuntary but not voluntary orienting contributes to a disengage deficit in visual neglect. Cortex, 46, 1149-1164.

Peterson, S.A., \& Gibson, T.N. (2011). Implicit attentional orienting in a target detection task with central cues. Consciousness and Cognition, 20, 1532-1547.

Pinheiro, J.C., \& Bates, D.M. (2000). Mixed-Effects Models in S and S-PLUS. New York: Springer-Verlag. doi:10.1007/b98882

Posner, M.I. (1980). Orienting of attention. Quarterly Journal of Experimental Psychology, 32, 325.

Prinzmetal, W., McCool, C., \& Park, S. (2005). Attention: Reaction Time and Accuracy Reveal Different Mechanisms. Journal of Experimental Psychology: General, 134, 73-92.

Prinzmetal, W., \& Landau, A.N. (2008). Dissecting spatial visual attention. In V. Coltheart Tutorials in visual cognition (pp. 43-66). Hove, UK: Psychology Press. 
R Development Core Team (2012). R: A language and environment for statistical computing. Vienna, Austria: R Foundation for Statistical Computing. Retrieved from http://www.r-project.org/ Rafal, R., \& Henik, A. (1994). The neurology of inhibition: Integrating controlled and automatic processes. In D. Dagenbach, \& T. H. Carr (Eds.), Inhibitory processes in attention, memory and language (pp. 1-51). San Diego, CA: Academic Press.

Rieth, C.A., \& Huber, D.E. (2013). Implicit learning of spatiotemporal contingencies in spatial cueing. Journal of Experimental Psychology: Human Perception and Performance, 39, 1165-1180. Risko, E.F., Blais, C., Stolz, J.A., \& Besner, D. (2008a). Covert orienting: A compound-cue account of the proportion cued effect. Psychonomic Bulletin \& Review, 15, 123-127.

Risko, E.F., Blais, C., Stolz, J.A., \& Besner, D. (2008b). Nonstrategic contributions to putatively strategic effects in selective attention tasks. Journal of Experimental Psychology: Human Perception and Performance, 34, 1044-1052.

Risko, E.F., \& Stolz, J. A. (2010a). The proportion valid effect in covert orienting: Strategic control or implicit learning? Consciousness and Cognition, 91, 432-442.

Risko, E.F., \& Stolz, J. A. (2010b). On the nature of cognitive control and endogenous orienting:

A Response to Chica and Bartolomeo (2010). Consciousness and Cognition, 19, 445-446.

Ristic, J. \& Kingstone, A. (2006). Attention to arrows: Pointing to a new direction. Quarterly Journal of Experimental Psychology, 59, 1921-1930.

Schneider, W., Eschman, A., \& Zuccolotto, A. (2002). E-Prime User's Guide. Pittsburgh: Psychology Software Tools, Inc.

Schmidt, J., (2013). Questioning conflict adaptation: proportion congruent and Gratton effects reconsidered. Psychonomic Bulletin \& Review, 20, 615-630.

Tassinari, G., Aglioti, S., Chelazzi, L., Marzi, C.A., \& Berlucchi, G. (1987). Distribution in the visual field of the costs of voluntarily allocated attention and of the inhibitory after-effects of covert orienting. Neuropsychologia, 25, 55-71. 
Tipples, J. (2002). Eye gaze is not unique: Automatic orienting in response to uninformative arrows. Psychonomic Bulletin \& Review, 9, 314-318.

Venables, W.N. (1998). Exegeses on linear models. Paper presented to the S- Plus User's Conference. Washington DC, October 8-9, 1998.

Vossel, S., Bauer, M., Mathys, C., Adams, R.A., Dolan, R.J., Stephan, K.E., \& Friston, K.J. (2014). Cholinergic stimulation enhances Bayesian belief updating in the deployment of spatial attention. Journal of Neuroscience, 19, 15735-15742.

Vossel, S., Mathys, C., Daunizeau, J., Bauer, M., Driver, J., Friston, K.J., \& Stephan, K.E. (2014). Spatial attention, precision, and Bayesian inference: a study of saccadic response speed. Cerebral Cortex, 24, 1436-1450.

Wansard, M., Bartolomeo, P., Vanderaspoilden, V., Geurten, M., \& Meulemans T. (2015).

Can the exploration of left space be induced implicitly in unilateral neglect?

Consciousness \& Cognition 31, 115-123.

Zhao, J., Al-Aidroos, N., \& Turk-Browne, N.B. (2013). Attention is spontaneously biased toward regularities. Psychological Science, 24, 667-677.

\section{Acknowledgements}

MB was funded by a FWO Pegasus/Marie Curie IEF Fellowship within the 7th framework program (Project 625378 "SpaceLOAD". GP is funded by the Special Research Fund from Ghent University and by the Belgian Science Policy, Interuniversity Attraction Poles program (P7/11). The authors are grateful to Marco Zorzi for providing access to lab facilities and to two anonymous reviewers for constructive comments on the manuscript. MB is grateful to Carlo Umiltà for valuable discussions on the study and to L. Naert for proofreading. 